\title{
THE SENSITIVITY OF THE MACROSCOPIC CONSEQUENCES OF VOID GROWTH IN DUCTILE MATERIALS TO VARIOUS MECHANICAL AND GEOMETRICAL MICRO-PARAMETERS
}

\author{
Li Guo Chen† \\ Institute of Mechanics, Academia Sinica, Beijing, China
}

and

\author{
I. C. HOWARD
}

Department of the Theory of Materials, Sheffield University, Mappin Street. Sheffield, S1 3JD, England

(Received 21 September 1982; in retised form 25 January 1983)

\begin{abstract}
The deformation of a cylindrical cell of elastic-plastic material containing an initially spherical void has been investigated in order to discuss the relative importance of the controlling mechanical and geometrical micro-parameters. If, as in this investigation, both strain hardening and strain softening occur in the matrix there are four main mechanical parameters. Two geometrical parameters complete the set of control variables. The individual influence of each of these variables and their interaction produce different overall responses of the cell, and this makes it possible to use macro-experimental data to calibrate the theoretical model.
\end{abstract}

\section{INTRODUCTION}

There is considerable experimental evidence (see, e.g. the work of Hancock and Mackenzie[1] and Lautridou and Pineau [2]) to suggest that, in steels at least, the macroscopic behaviour of the material at high plastic strain or triaxial stress is influenced by the generation of second order holes other than those associated with the largest particles or inclusion in the material. As an element of material is subjected to increasing strain and stress, voids initiate and grow around the large MnS particles. The growth of these voids and the effect of the growth on the behaviour of the element as a whole has occupied the attention of most workers on the ductile failure of materials. However, there are smaller objects (the fine carbides and other precipitates) that form the nucleation sites of finer voids, the nucleation taking place if the strain and/or the triaxial stress is large enough. The growth of these fine voids will soften the matrix of the cell in exactly the same way as the cell itself is softened by the growth of the larger voids associated with the $\mathrm{MnS}$ particles. This softening of the matrix accelerates the growth of the main voids and lowers the stress and strain at which the initiation of fracture is predicted by any model of their growth and coalescence.

We have already presented [3] a detailed discussion of theoretical analyses of void growth in ductile materials, and the interested reader may refer to that paper. However, in setting the perspective for the work reported here it is useful to point out that most authors have chosen to discuss one or the other of the following three alternatives; plane strain or axisymmetric deformation, perfectly plastic or plastically hardening material, and a simplified or detailed account of the interaction enforced by the containing material on the boundary of the cell.

We have decided that an axisymmetric cell is particularly versatile for the study of the effects of void growth within general patterns of continuum deformation, that it is important to compute with definite hardening characteristics (and our results are for a material that hardens in such a way that it correctly simulates the necking behaviour of A533B nuclear pressure vessel steel in a simple unixial test), and that the study of the whole range of possible interactions with the containing medium is vital to the application of our results to various real ductile fractures.

tName in Chinese phonetic spelling with surname first. The work on which this paper is based was completed whilst the first named author was visiting the Department of Mechanical Engineering, Sheffield University, England. 
Within this framework our efforts have been directed towards evaluating the effects of the microstructural parameters that affect the straining and instability of the model cell. This has been achieved by performing a large number of computations that develop the relationships between the macroscopic response of the cell and the micro-parameters that control it. Of course, some advance towards this goal has already been made by other workers in this field, but it is almost impossible to discuss the effect we seek in that work because of the differences in modelling that we have already alluded to. Our work is an attempt at a truly systemmatic analysis of the problem.

\section{THE COMPUTATIONAL MODEL}

We have investigated the response of a voiding elastic-plastic continuum by studying the deformation of a cylindrical cell of matrix material which contains an initially spherical void. A quadrant of the cell is shown in Fig. 1 . The deformation of the cell was analysed by subdividing it into 456 constant strain triangular finite elements with 260 nodes, and the large strains involved were computed by the up-dated Lagrangian formulation as set out by McMeeking and Rice [4].

Our intention was to investigate the quantities associated with the overall, or macroscopic response of the cell, the fields of stress and deformation within the cell and the mechanical geometrical parameters that control them. We shall, where appropriate, use the prefix "macro" to denote the response of the cell as a whole and "micro" to denote the variable fields within the cell.

The value of the macroscopic axial true stress $\overline{\sigma_{z}}$ is found by adding the nodal forces along the top horizontal surface and dividing the result by the current area of the top of the cell. The corresponding radial stress $\bar{\sigma}_{r}$ is found in an analogous way. At the beginning of each increment of loading the boundaries of the cell are moved to the position indicated by the displacement computed during the previous increment. Macroscopic strains are

$$
\begin{aligned}
& \overline{\epsilon_{z}}=\ln \left(1+\Delta L_{0} / L_{0}\right) \\
& \overline{\epsilon_{r}}=\ln \left(1-\Delta R_{0} / R_{0}\right) .
\end{aligned}
$$

We regulate the change of shape of the cell through the value of the constant $\alpha$ which relates the current values of the macro-strain rates by

$$
\dot{\bar{\epsilon}}_{r}=-\alpha \dot{\bar{\epsilon}}_{:}\left(\text {i.e. } \frac{\dot{R}}{R}=-\alpha \frac{\dot{L}}{L}\right)
$$

This integrates to

$$
\bar{\epsilon}_{r}=\alpha \overline{\epsilon_{i}}
$$

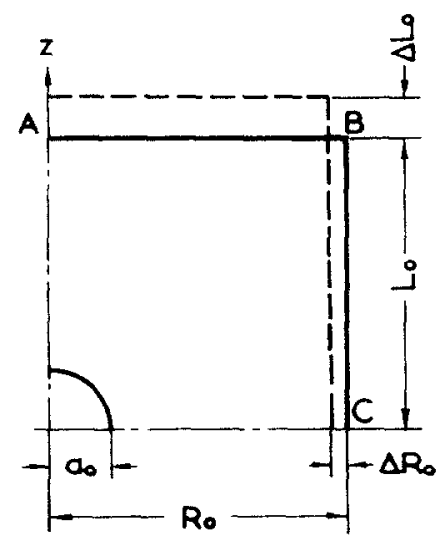

Fig. 1. A quadrant of the axisymmetric unit cell used to model the growth of a void. 
which is the condition of macro-proportional straining. It should yield

$$
\frac{\Delta R_{0}}{R_{0}}=1-\exp \left(-\alpha \bar{\epsilon}_{z}\right)
$$

Deviation from proportional straining would occur if the loading displacement increments were too big. Small increments of loading are therefore desirable.

Different overall dilatations of the cell can be simulated by changing the value of $\alpha$. The following macro-parameters will be used to discuss the behaviour of the cell.

$$
\begin{aligned}
& \overline{\sigma_{e}}=\overline{\sigma_{z}}-\overline{\sigma_{r}} \text { is the equivalent stress } \\
& \overline{\epsilon_{e}}=\frac{2}{3}\left(\overline{\epsilon_{z}}-\overline{\epsilon_{r}}\right)=\frac{2}{3}(1+\alpha) \overline{\epsilon_{z}} \text { is the equivalent strain } \\
& \overline{\sigma_{m}}=\left(\overline{\sigma_{z}}+2 \overline{\sigma_{r}}\right) / 3 \text { is the mean stress } \\
& \overline{\epsilon_{m}}=\left(2 \bar{\epsilon}_{r}+\bar{\epsilon}_{z}\right) / 3=(1-2 \alpha) \overline{\epsilon_{z}} / 3 \text { is the mean strain } \\
& \frac{\dot{\omega}}{\omega \bar{\epsilon}_{e}} \text { is a nomalized void growth parameter, where } \omega \text { is the current void volume. }
\end{aligned}
$$

current void volume.

We assume that softening begins in the matrix when

$$
\sigma_{c y}=\left(\sigma_{m}+\lambda_{e} \sigma_{e}\right) / \bar{\sigma}_{y}
$$

where $\bar{\sigma}_{v}$ is the yield stress of the material, $\sigma_{c y}$ and $\lambda_{e}$ are two material constants and $\sigma_{m}$ and $\sigma_{e}$ are the mean stress and equivalent stress in the matrix. (This criterion has the same functional form as that proposed by Lautridou and Pineau[2] for the nucleation of voids, but our material constants will be different from theirs unless the nucleation of the voids and the softening due to their growth are close together.) In the computations the normalized elongation $\Delta L_{0} / L_{0}$ is taken to be the generalized time $t$. The tangent modulus used in the Prandtl-Reuss flow theory is defined as ${ }^{\dagger}$

$$
E_{\mathrm{t}}=\frac{\mathscr{D} \sigma_{e}}{\mathscr{D} t} / \dot{\boldsymbol{\epsilon}}_{e}
$$

where $\mathscr{D} \sigma_{e} / \mathscr{D} t$ is the Jaumann derivative of the equivalent stress, and the rate of equivalent strain is

$$
\dot{\epsilon}_{e}=\frac{2}{3}\left(\dot{\epsilon}_{z}-\dot{\epsilon}_{r}\right)=\frac{2}{3}(1+\alpha) \dot{\epsilon}_{z}
$$

We characterize the hardening of the cell by the macroscopic tangent modulus

$$
\bar{E}_{t}=\frac{\mathscr{D} \bar{\sigma}_{e}}{\mathscr{D} t} / \dot{\bar{\epsilon}}_{e},
$$

the actual values used in the computations being given in Table 1, and define the parameters

$$
\beta_{t}=E_{l} / \bar{E}_{\mathrm{t}} \text { and } e_{f}=E /\left(-E_{f}\right)
$$

$\left(-E_{f}\right)$ is the (negative) softening modulus of the matrix material which replaces $E_{t}$ when condition (5) is satisfied. $\ddagger$ Four material parameters, $\sigma_{c y}, \lambda_{e}, \beta_{t}$ and $e_{f}$ have now been identified.

tThe difference between total strain and plastic strain is neglected in this definition.

\$The assumption of a constant, negative tangent modulus when the softening point of the matrix has been reached is the simplest way of dealing with the phenomenon. There are, of course, more detailed approaches available, in particular those based on the models of Gurson[5]. For comparison, Tvergaard[6], using Gurson's model, requires three parameters to characterize the softening of the material. The discussion of effects of the detailed characteristics of the softening of the matrix on the response of the cell is reserved for a future study. 
Table 1. The variation of the tangent modulus $\bar{E}_{t}$ with the strain $\bar{\epsilon}_{e} . \bar{E}=207 \mathrm{GPa}, \bar{\nu}=0.29$ and $\bar{\sigma}_{y}=458 \mathrm{MPa}$

\begin{tabular}{|c|c|c|c|c|c|c|}
\hline $\bar{E}_{\bar{E}}$ & 0.010 & 0.030 & 0.040 & 0.650 & U. OE, & 0.680 \\
\hline $\bar{F}^{\prime}, \bar{E}_{t}$ & 68 & 76 & 90 & 20 & 14 & 194 \\
\hline$\vec{\varepsilon}_{\mathrm{e}}$ & 0.100 & 0.120 & 0.140 & 0.500 & 0.300 & 1.10 \\
\hline$\overline{\mathrm{E}}, \overline{\mathrm{E}}_{\mathrm{t}}$ & 236 & 340 & 440 & $\$ 50$ & 460 & 470 \\
\hline$\vec{E}, \equiv_{t}$ & +40 & 1.10 & $\vec{E}_{0}$ & & & \\
\hline
\end{tabular}

Two more

$$
r_{0}=\frac{a_{0}}{R_{0}} \text { and } \rho=\frac{L_{0}}{R_{0}}
$$

complete the mechanical and geometrical specification of the cell.

When the overall axial stress $\bar{\sigma}_{z}$ reaches a peak and begins to drop, the cell is understood to have reached its point of instability.

This definition of the failure of the cell was also used by Rousselier [7]. It may, however, be preceded by the development of a shear band, or, as predicted by Tvergaard [8], be followed by it. In either case the macroscopic stress at which the instability point of the cell and the formation of a shear band occur are close.

The computations were performed so that the macroscopic tangent modulus $\vec{E}_{\mathrm{t}}$ agreed with that simulated by Li[9] for the tensile deformation of A533B steel. The computational method was described in detail by $\mathrm{Li}$ and Howard [3].

\section{THE MECHANICAL PARAMETERS OF THE MATRIX}

The overall stress-strain curves of the cell, its instability point and the value of the void growth parameter at the instability point vary with the values chosen for the mechanical parameters of the matrix.

Figure 2 shows the effects of $\sigma_{c y}$ and $\beta_{t}$ on the stress-strain curves with $\lambda_{e}, e_{f}, r_{0}$ and $\rho$ kept constant. Different values of $\sigma_{c y}$ significantly influence the peak values of the equivalent stress $\bar{\sigma}_{e}$ and the mean stress $\bar{\sigma}_{m}$. The slopes of the stress-strain curves are mainly affected by the values of $\beta_{t}$. On the other hand, lowering $\sigma_{c y}$ has the effect of increasing the void growth parameter and simultaneously decreasing the triaxiality parameter at instability as shown by the data represented by solid triangles and squares in Figs. 3 and 4 . Comparing the blank triangular data with those of the solid squares, we can see that the hardening ratio $\beta_{t}$ has the effect of lowering the slope of the void growth parameter curve in Fig. 3, whilst raising the slope of the triaxiality curve in Fig. 4.

The effects of changing $\lambda_{e}$ are shown in Fig. 5 where $\sigma_{c v}, \beta_{1}, e_{f}, r_{0}$ and $\rho$ are kept constant. The value of $\lambda_{e}$ has a strong influence on the maximum value of $\sigma_{e}$ but its effect on the mean stress-strain curve is slight. A comparison of Fig. 2 in which $\epsilon_{f}=-100$ with Fig. 5 in which $e_{f}=-50$ shows that the effect of $\epsilon_{f}$ on the hardening of the cell is almost imperceptible whilst the slope of the softening part of the curves varies directly with $e_{f}$, as one might have expected. Further information on the effects of changes in $\lambda_{e}$ can be obtained from an examination of the data represented by blank squares and inverted blank triangles in Figs. 3 and 4. Variation of $\lambda_{e}$ has no significant influence on the void growth rate locus at instability and it affects the triaxiality condition only in the lower triaxiality range $\left(\bar{\sigma}_{m} / \bar{\sigma}_{e} \leqq 2\right)$. However, a comparison of the data represented by blank and solid squares shows that the softening parameter $\epsilon_{f}$ significantly influences both of these relationships; an increase of the absolute value of $e_{f}$ lessens the void growth parameter at instability (Fig. 3) and strengthens the triaxiality (Fig. 4).

The triaxiality of the cell is controlled by the value of $\alpha$. However, in many applications it is the value of the stress triaxiality $\bar{\sigma}_{m} / \bar{\sigma}_{e}$ that is of direct interest as a control variable on the 

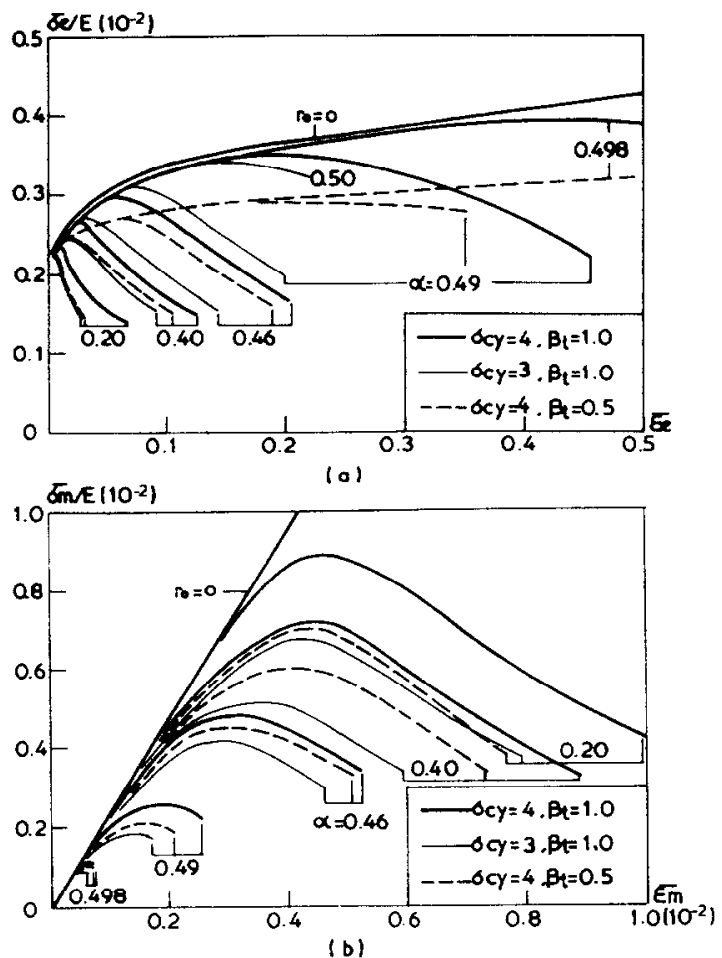

Fig. 2. The effect of $\sigma_{c y}$ and $\beta_{1}$ on the stress-strain curves. $\left(\lambda_{e}=1.67 . e_{f}=-100, r_{0}=0.2, \rho=1\right)$.

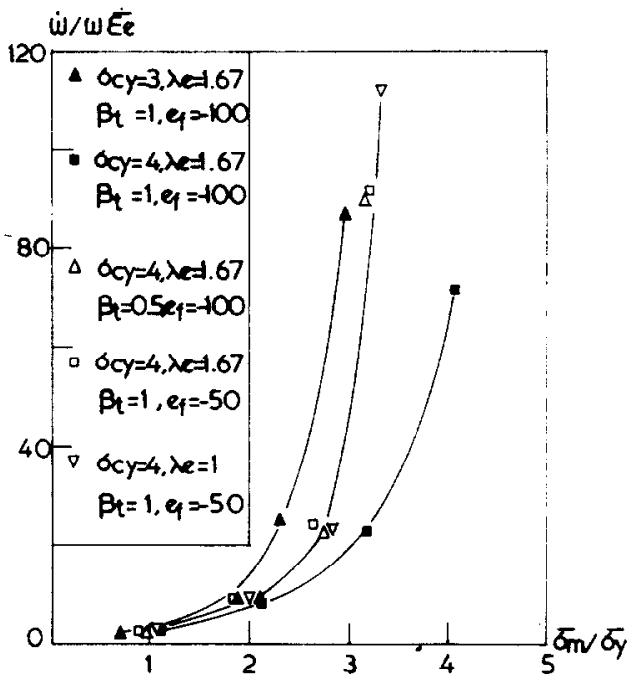

Fig. 3. The void growth parameter $\dot{\omega} / \omega \dot{\bar{\epsilon}}_{i}$ vs the normalized mean stress $\bar{\sigma}_{m} / \bar{\sigma}_{y}$ at the instability point for various mechanical parameters of the cell $\left(r_{0}=0.2 . \rho=1\right)$.

behaviour of the cell, and the relationship between this and $\alpha$ may be of interest to those who wish to use our results. The relationship we seek may be read from Figs. 3 and 4 when one adds to those the fact that the data are computed for $\alpha$ values of $0.2,0.4,0.46$ and $0.49, \alpha=0.2$ corresponding to the highest triaxiality. It is immediately apparent that, except for the lowest degree of triaxiality, the relationship between $\bar{\sigma}_{m} / \bar{\sigma}_{e}$ and $\alpha$ is sensitive to the value of $\sigma_{c y}$. The general trend is clear though, in that the triaxiality increases as $\alpha$ decreases, but the spread in $\bar{\sigma}_{m} / \bar{\sigma}_{e}$ due to different values of $\sigma_{c y}, \lambda_{e}, \beta_{t}$ and $e_{f}$ increases with triaxiality. Figure 4 shows that $\alpha=0.2$ produces leveis of hydrostatic tension that are consistent with what is believed to occur 


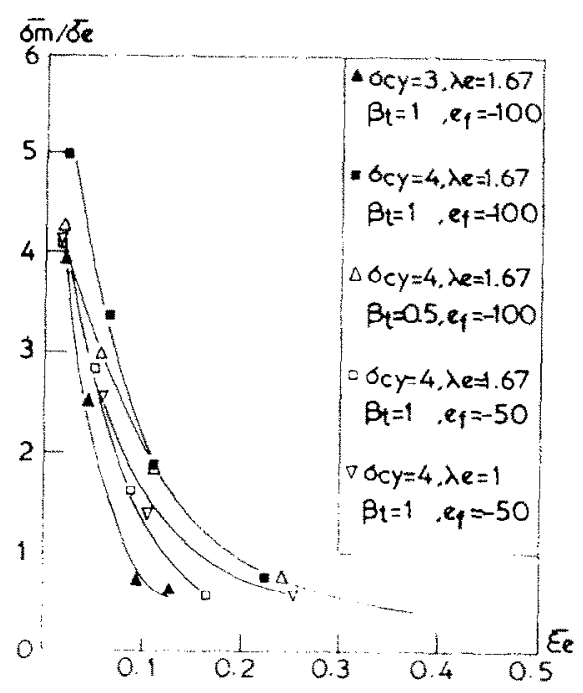

Fig. 4. The triaxiality parameter $\bar{\sigma}_{m} / \bar{\sigma}_{e}$ vs the equivalent strain $\bar{\epsilon}_{e}$ (at the instability point) for various mechanical parameters of the cell $\left(r_{0}=0.2 . p=11\right.$.
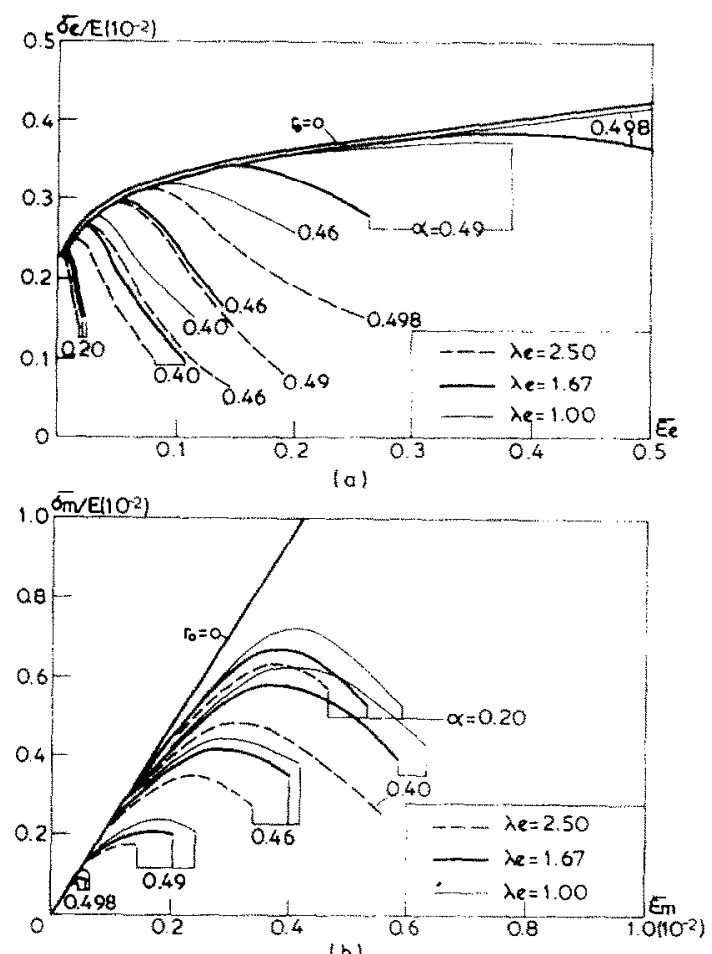

(b)

Fig. 5. The effects of $\lambda_{e}$ on the stress-strain curves $\left(\sigma_{t y}=4, \beta_{t}-1, e_{f}=-50, r_{0}=0.2, \rho=1\right)$

near crack tips, (computed values [10] for the maximum stress near a crack tip in a material with a hardening index between 0.1 and 0.2 are between 3 and 4 times $\sigma_{y}$ as is the data of Fig. 4 when $\alpha=0.2$ ) whilst the data at high $\alpha$ levels would correspond to the behaviour in necking bars. This observation points to the importance of microstructural parameters in characterizing the highly triaxial events close to crack tips, and illustrates the potential dangers of using data collected on plain tensile specimens to predict the behaviour of ductile cracks. 
THE GEOMETRICAL PARAMETERS OF THE MODEL CELL

One might expect that enlarging the initial size of the void would soften the response of the cell and this has been demonstrated previously in Figs. 10 and 11 of Li and Howard [3]. The effect is shown again in Fig. 6(a) of this paper in the bodily drop of the whole curve for the case $\lambda_{e}=1.67$, with the resulting halving of the strain to instability as $r_{0}$ is doubled with a wide range of $\lambda_{e}$.

Also shown in Fig. 6(a) by the data on the broken lines is the result of changing $\lambda_{e}$ whilst keeping everything else fixed. The hardening part of the curve is unaffected, but there is a dramatic change in the softening part and the instability point over the range of $\lambda_{e}$ for which computations have been made. Figures 7 and 8 show (amongst other things) how the void growth parameter at instability and the triaxiality state are affected by a change in $r_{0}$. The data represented by the thin solid line and the broken line show that there is a large drop in the triaxialty curve as $r_{0}$ is doubled and, for the same value of $\overline{\sigma_{m}}$, the instability void growth parameter increases (due to the decrease of ductility) at instability.

The results of varying the values of $\rho$ are also shown in Figs. 6-8. There is little change of any of the parameters.

\section{DISCUSSION}

We have studied the sensitivity of the overall mechanical response and the pattern of void growth in the cell to the micro-mechanical parameters $\sigma_{c y}, \lambda_{e}, \beta_{i}$ and $e_{f}$ of the matrix and the geometrical parameters $r_{0}$ and $\rho$. The main effects of increasing each of these six parameters are summarized in Table 2.

Within the range of values chosen for the computations the influence of the initial length-radius ratio $\rho$ can be neglected. The effect of increasing $r_{0}$ is, in many respects, similar to that of decreasing $\sigma_{c y}$, a fact that may make $\sigma_{c y}$ a particularly useful parameter in simulating ductile failure. Table 2 shows that $\lambda_{e}$ measures the influence of the equivalent stress $\bar{\sigma}_{e}$. Furthermore, it can adjust the ratio between the overall axial stress $\bar{\sigma}_{z}$ and the overall radial stress $\bar{\sigma}_{n}$, since it strongly affects the peak value of $\bar{\sigma}_{e}$ whilst it has only moderate influence on $\bar{\sigma}_{m}$. When $\bar{\sigma}_{m} / \bar{\sigma}_{e}<2$ it can be used to adjust the curvature of the $\bar{\sigma}_{m} / \bar{\sigma}_{e}-\bar{\epsilon}_{e}$ relationship of Fig. 4.
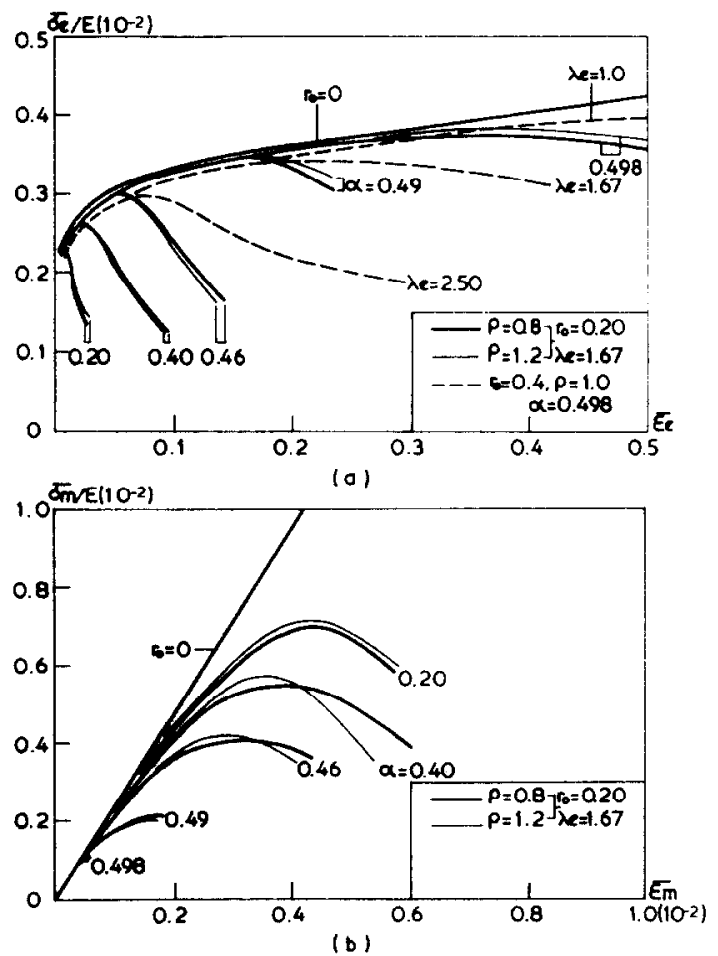

Fig. 6. The effects of $r_{0}$ and $\rho$ on the stress-strain curves $\left(\sigma_{c y}=4, \beta_{t}=1, e_{f}=-50\right)$. 


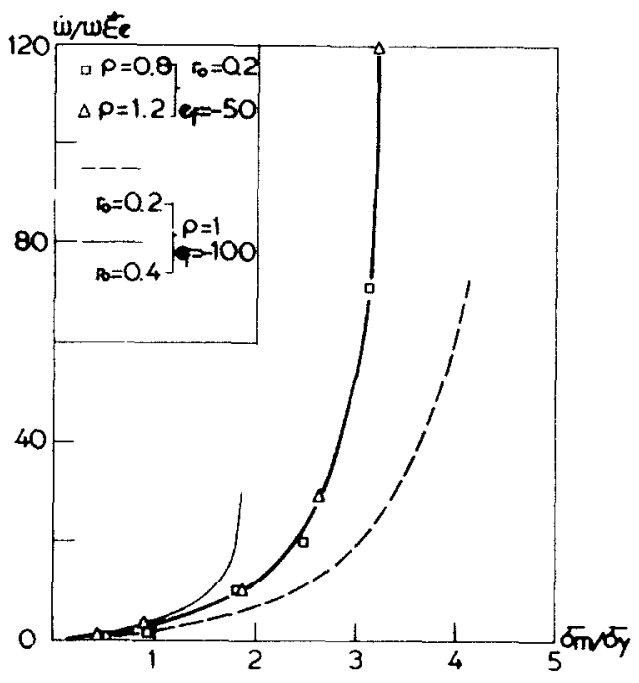

Fig. 7. The void growth parameter $\dot{\omega} / \omega \dot{\bar{\epsilon}}_{e}$ vs the normalized mean stress $\bar{\sigma}_{m} / \dot{\sigma}_{y}$ (at the instability point) for various geometrical parameters of the cell $\left(\sigma_{c y}=4, \lambda_{e}=1.67, \beta_{t}=1\right)$.

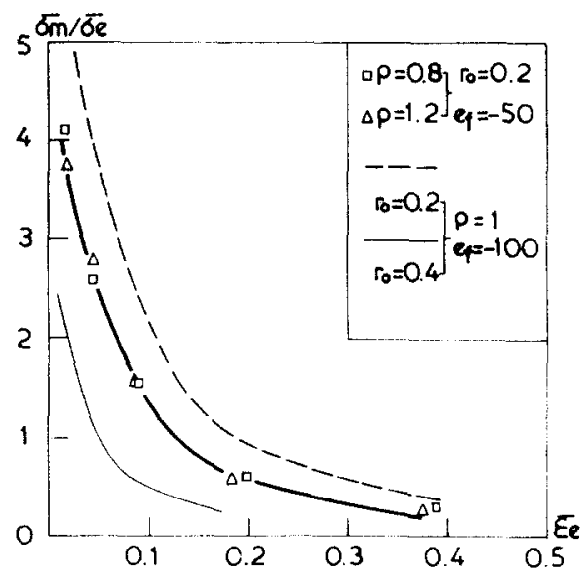

Fig. 8. The triaxiality parameter $\bar{\sigma}_{m} / \bar{\sigma}_{\mathrm{e}}$ vs the equivalent strain $\bar{\epsilon}_{e}$ (at the instability point) for various geometrical parameters of the cell $\left(\sigma_{c v}=4, \lambda_{e}=1.67, \beta_{t}=1\right)$.

The observations suggest that the choice of values for the four mechanical parameters $\sigma_{c y}$, $\lambda_{e}, \beta_{t}, e_{f}$ is of the most importance for correctly simulating the continuum response of this void model. The influence of $r_{0}$ overlaps that of $\sigma_{c y}$ in many respects especially for high strength steels. The effects of $\rho$ are negligible.

All this points to the possibility of simulating the parameters of the void model by making use of the experimental data of necking and notched bars. The determination of $\sigma_{c y}$ depends on the fact that the continuum softening point should be somewhere near the point of void coalescence or the beginning of stable crack growth. The experimental techniques needed for testing void nucleation and void coalescence have been resolved by the Beremin group [11] and Hancock et al.[1, 12]. The values of $\beta_{t}$ and $e_{f}$, which are related to the continuum tangent modulus $\bar{E}_{t}$, can be determined by computer simulation of a necking bar $[9,13]$. This is accomplished by adjusting the stress-strain response of the material until the computed response of the bar agrees with the experimental data. A series of notched bar tests [1,2] gives the locus between the triaxiality parameter $\bar{\sigma}_{m} / \bar{\sigma}_{e}$ and the equivalent strain $\bar{\epsilon}_{e}$ at the initiation of failure. The relationship of $\bar{\sigma}_{m} / \bar{\sigma}_{e}-\bar{\epsilon}_{e}$ helps to select an appropriate value for $\lambda_{e}$. Finally, the value of $r_{0}$ can be chosen from the fact that a change in it moves the overall equivalent stress-strain curve bodily up or down. 
Table 2. The effects of increasing the values of the characterizing parameters on various relationships

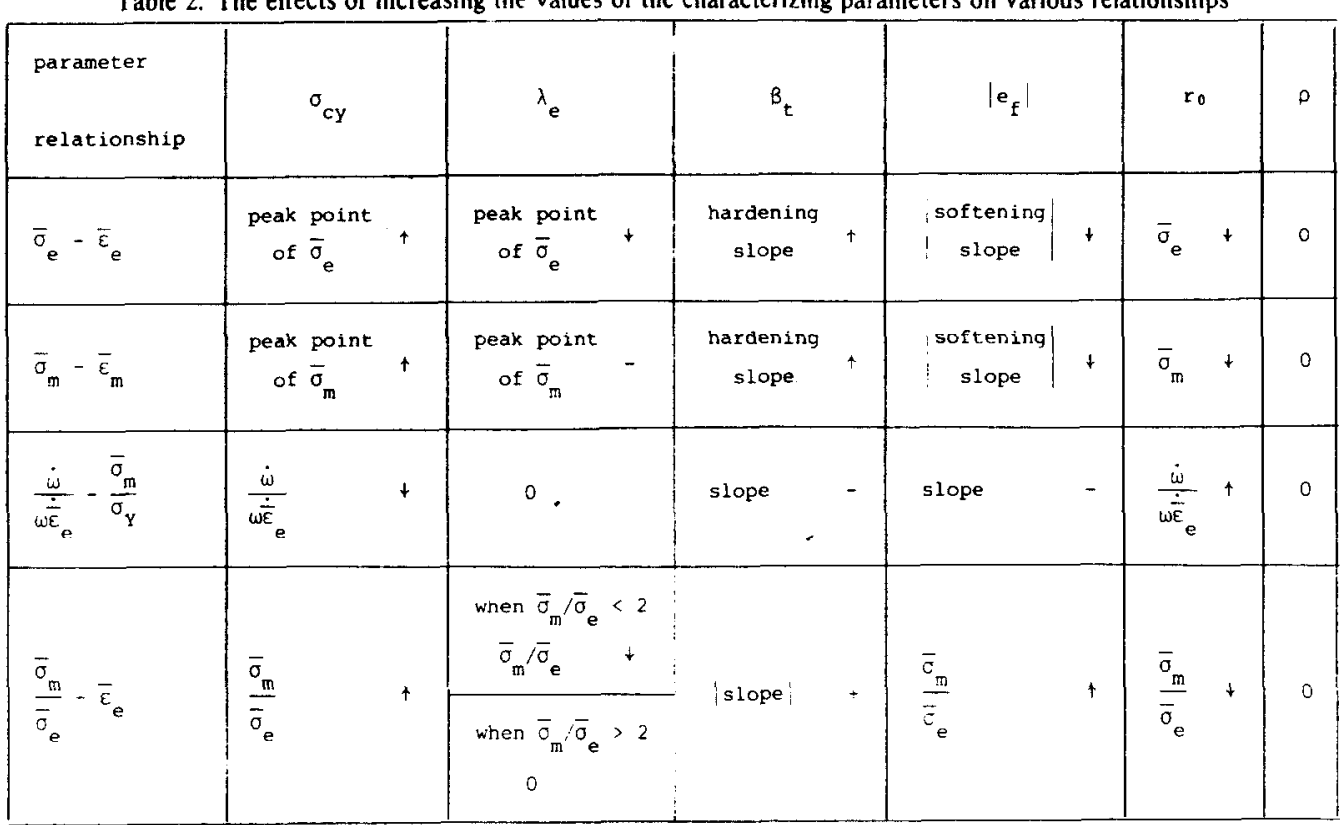

Notation:

$\uparrow$ significantly increases, $\downarrow$ significantly decreases, 0 very sight influence

+ moderately increases, - moderately decreases, $\quad$ | absclute value

Of course, some "trial and error" tests have to be performed at various stages of any complete simulation. A stringent test of a simulation would then be a comparison between the computed result and the information obtained from metallographic examination of the material.

Even so, any computed result is still approximate because the computer simulations of necking bars have only been done with the assumption of plastic incompressibility; the dilational effect in plasticity has not yet been taken into account. This inaccuracy will be lessened, if not eliminated, once the dilatational response predicted by the calibrated cell is incorporated into the constitutive equations of the continuum that represents the material in the necking bar. The implementation of the ideas outlined above are underway and will form the subject of a further paper.

Some connection with experimental observations can be made by comparing our Figs. 4 and 8 with Fig. 3 of Lautridou and Pineau[2]. Their work was on A508 steel at $100^{\circ} \mathrm{C}$ and the similarity between that material and the AF33B approximately simulated here should be enough for a sensible comparison to be made. They used four steels, designated $A, B, C$ and $D$, tested variously in the long $(L)$, transverse $(T)$ and short $(S)$ rolling directions. The effects of this were

Table 3. The parametric dependence of the relationship $\dot{a} / a=\gamma \dot{\bar{\epsilon}}_{e} \exp \left(\frac{3}{2} \bar{\sigma}_{m} / \bar{\sigma}_{e}\right)$ at the point of instability

\begin{tabular}{|cccccc|}
\hline$\alpha$ & $x_{0}$ & $\dot{a} / \alpha$ & $\dot{\bar{\varepsilon}}_{\mathrm{e}}$ & $\bar{\sigma}_{\mathrm{m} / \bar{\sigma}_{\mathrm{e}}}$ & $Y$ \\
\hline \multirow{2}{*}{0.40} & 0.10 & 0.083 & 0.0042 & 6.0 & 0.0023 \\
& 0.20 & 0.051 & 0.0069 & 3.3 & 0.048 \\
& 0.40 & 0.010 & 0.0070 & 1.0 & 0.32 \\
\hline \multirow{2}{*}{0.45} & 0.10 & 0.068 & 0.0066 & 6.0 & 0.0012 \\
& 0.20 & 0.051 & 0.014 & 2.9 & 0.047 \\
& 0.40 & 0.0054 & 0.0072 & 0.56 & 0.33 \\
\hline
\end{tabular}


mainly to sample different sizes and projected shapes of the inclusions, which were either cllipsoidal $(A$ and $D)$ or disc shaped ( $B$ and $C$ ). Steel $A$ was tested in the $L$ and $S$ orientations, the $S$ orientation producing data that lie within the lower part of the curves of our Fig. 4 . Their data for steel $A$ in the $L$ orientation are, however, somewhat above our curves. Figures 4 and 8 and Table 2 show that an eleyation of the ductility curve is associated with increasing $\sigma_{c y}$ or $\left|e_{f}\right|$ or a decrease in $r_{0}$. It is likely that $r_{0}$ is the controlling parameter as $\sigma_{c y}$ and $\left|e_{f}\right|$ are associated only with the properties of the matrix. If this so, the effective value of $r_{0}$ is related to the average diameter of the inclusion perpendicular to the axis of the test, the diameter in the $L$ orientation being smaller than that in the $S$ orientation and results supporting this view have been obtained on a study [14] of the effect of void shape on growth. Figure 8 now shows how the ductility curve is projected bodily upwards as $r_{0}$ decreases, as the experiments suggest. This effect of orientation appears to be more significant than that of volume alone, for steel $A$ had bigger particles than steel $D$ with about twice the volume fraction of inclusions but the ductility curves for these two materials in the $L$ orientation are virtually indistinguishable. The effect is also shown in the work of Hancock and Mackenzie [1] who present qualitatively similar data on orientation effects in Q1 and HY130 steels.

The role of triaxiality in pronoting void growth is often expressed through a relationship of the form $[15,16]$

$$
\frac{\dot{a}}{a}=\gamma \bar{\epsilon}_{e} \exp \left(\frac{3}{2} \frac{\bar{\sigma}_{m}}{\bar{\sigma}_{e}}\right)
$$

Table 3 (mainly taken from data published elsewhere [3] and reproduced here in Fig. 3) shows how this relationship at the point of instability is affected by changes in some of the control parameters. The most striking feature is the extremely rapid growth of the pre-exponential term $\gamma$ with $r_{0}$, a prediction that agrees qualitatively with experimental observation. However, the variation predicted here seems to be much larger than that in the experiments[17]. This may have something to do with the non-spherical shape of many particles in real materials, but this disparity will probably not be resolved until a programme of simultaneous testing and computer simulation has taken place.

\section{REFERENCES}

1. J. W. Hancock and A. C. Mackenzie, On the mechanisms of ductile failure in high-strength steels subjected to multi-axial stress states. J. Mech. Phys. Solids 24, 147 (1976).

2. J. C. Lautridou and A. Pineau, Crack initiation and stable crack growth resistance in A508 steets in relation to inclusion distribution. Engng Fracture Mech. 15, 55 (1981).

3. Li Guo Chen and I. C. Howard, The effect of strain softening in the matrix material during void growth. J. Mech. Phys. Solids 31, 85 (1983).

4. R. M. McMeeking and J. R. Rice, Finite-element formulations for problems of large elastic-plastic deformation. Imt. J. Solids Structures 11, 601 (1975).

5. A. L. Gurson, Continum theory of ductile rupture by yoid nucleation and growth: Part 1-yield criteria and flow rules for porous ductile media. I. Engng Mat. Technol. 99, 2 (1977).

6. V. Tvergaard, Ductile fracture by cavity nucleation between larger voids J. Mech. Phvs. Solids 30, 265, (1982).

7. G. Rousselier, Constitutive relations including ductile fracture damage. Application to cracked bodies. Adrances in Fracture Research (Edited by D. Francois), ICF5. Cannes, France, 29 March-3 April 1981, Vol. 2, p. 803. Pergamon Press, Oxford (1981).

8. V. Tvergaard, On localization in ductie materials containing spherical voids. Iht I. Fracture 18, 237 (1982).

9. Li Guo Chen, Necking in uniaxial tension. Int. J. Mech. Sci. 25, 47 (1983)

10. R. M. McMeeking. Finite deformation analysis of crack-tip opening in elastic-plastic materials and implications for fracture. J, Mech. Phys. Solids 25, 357 (1977).

11. F. M. Beremin, Cavity formation from inclusions in ductile fracture of A508 steel. Met. Trans. A 12A. 723 (1981)

12. J. W. Hancock and M. J. Cowling, Role of State of Stress in crack tip failure processes. Metal Sci. 14, 293 (1980).

13. D. M. Norris, Jr., B. Moran, J. K. Scudder and D. F. Quinones, A computer simulation of the tension test. J. Mech. Phys. Solids 26, 1 (1978).

14. Li Guo Chen, Unpublished work (1983).

15. F. A. McClintock, A criterion for ductile fracture by the growth of holes. J. Appl. Mech., 35, 363 (1968).

16. J. R. Rice and D. M. Tracey, On the ductile enlargement of voids in triaxial stress fields. J. Mech. Phys. Sollds 17, 201 (1969).

17. F. Mudry and A. Pineau, Private communication (1982). 